\section{Comorbidade entre depressão e doenças clínicas em um ambulatório de geriatria}

\author{
Depression and clinical illness: comorbidity in a \\ geriatric outpatient clinic
}

\author{
1 Faculdade de Medicina, \\ Universidade Federal da \\ Bahia, Salvador, Brasil. \\ Correspondência \\ M. B. Duarte \\ Departamento de Medicina \\ Preventiva, Faculdade de \\ Medicina, Universidade \\ Federal da Bahia. \\ Av. Reitor Miguel Calmon $s / n$ \\ Salvador, $B A$ \\ 40110-100, Brasil. \\ meirelayne@reim.com.br
}

\begin{abstract}
The aim of this study was to investigate the association between depression and clinical illness among a group of elderly in the city of Salvador, Bahia, Brazil. The study population included 1,120 individuals over 60 years of age treated at a geriatric outpatient clinic. Crude and stratified prevalence ratios were calculated. Data were stratified by gender, age, and nutritional status. Ninety-five percent of the sample presented at least one chronic disease, the most frequent being hypertension (62.2\%), osteoarthritis (40\%), and urinary incontinence (35\%). Depression was diagnosed in $23.4 \%$, more commonly among women ( $P R=1.28$; 95\%CI: 0.99-1.65) and those under 75 years $(P R=1.24 ; 95 \% C I$ : 1.00-1.53). There was an association between number of chronic diseases (> 3) and depression (PR = 1.31; 95\%CI: 1.04-1.66). Parkinson's disease was associated with depression, more evident among females ( $P R=1.59$; 95\%CI: 1.05$2.41)$ and in the 70-79-year age group $(P R=2.02$; 95\% CI: 1.28-3.20). The study demonstrates an association between depression and chronic comorbidity. Since many elderly present multiple chronic diseases, health professionals should be alert to the possibility of depressive symptoms in these patients.
\end{abstract}

Aged; Aging Health; Chronic Disease; Depression
Meirelayne Borges Duarte 1

Marco Antônio Vasconcelos Rego 1

\section{Introdução}

O envelhecimento populacional é um dos fenômenos mais notórios dos tempos atuais, em todo o mundo, trazendo consigo repercussões culturais, sociais e políticas 1 . O Brasil é um país que envelhece velozmente: a expectativa de vida aumentou de 33 para 68 anos durante o século XX 2,3. De acordo com a última Pesquisa Nacional por Amostra de Domicílios (PNAD 2004), a população de idosos ultrapassa 17 milhões, correspondendo a aproximadamente $10 \%$ da população brasileira 4 . As projeções para o ano 2020 estimam 32 milhões, o que colocará o Brasil na sexta posição mundial em número de idosos 5 .

$\mathrm{O}$ aumento progressivo na expectativa de vida implica aumento da morbidade por doenças crônicas não-transmissíveis, que muitas vezes são incapacitantes e que são determinantes da maior parte dos gastos com a saúde nos países desenvolvidos 6,7. A depressão na população idosa, por exemplo, é um importante problema de saúde pública em virtude de sua alta prevalência, freqüente associação com doenças crônicas, impacto negativo na qualidade de vida e risco de suicídio. Aproximadamente $15 \%$ a $20 \%$ dos idosos não institucionalizados apresentam sintomas depressivos 8 .

A comorbidade entre doenças físicas e mentais é de grande interesse, sendo geralmente aceito que a presença de uma patologia orgânica aumenta o risco de transtornos psiquiátricos 4 . 
Doenças clínicas podem contribuir para a patogênese da depressão através de efeitos diretos na função cerebral ou através de efeitos psicológicos ou psicossociais 9 .

Tal associação pode ser vista de modo bidirecional: a depressão precipitando doenças crônicas e as doenças crônicas exacerbando sintomas depressivos. Essa complexa relação tem implicações importantes tanto para o manejo das doenças crônicas, quanto para o tratamento da depressão 10 .

O principal objetivo deste estudo foi investigar a associação entre doenças crônicas e depressão em idosos de um ambulatório de referência, em Salvador, Bahia. Mais especificamente, o trabalho buscou avaliar a prevalência de depressão e de doenças crônicas em idosos e as possíveis diferenças entre os sexos; investigar associação entre depressão e as patologias crônicas estudadas; identificar diferenças por gênero e por faixa etária na associação entre depressão e doenças crônicas.

\section{Método}

\section{População de estudo}

O trabalho foi realizado em um ambulatório geriátrico de referência, em Salvador, Bahia, Brasil. A amostra de conveniência foi constituída pelos pacientes atendidos em consulta inicial pela autora do trabalho, no período de agosto de 2001 a outubro de 2004, totalizando 1.120 idosos. Não existe qualquer critério para o encaminhamento dos pacientes para um ou outro geriatra do centro, exceto a disponibilidade de vaga para aprazamento.

\section{Coleta de dados}

Os dados foram coletados durante a assistência aos pacientes, por meio de anamnese padronizada (realizada por enfermeiras treinadas), exame físico completo (realizado pela médica autora do trabalho) e exames complementares (glicemia, colesterol total, HDL, LDL, triglicérides, TSH, radiografia de tórax e ECG).

\section{Avaliação do estado nutricional}

Mediante cálculo do índice de massa corporal (IMC), os pacientes foram classificados em desnutridos (IMC < 22), eutróficos (IMC entre 22 e 27) obesos (IMC > 27), conforme classificação sugerida pela Organização Mundial da Saúde (OMS) para idosos.

\section{Critérios diagnósticos de depressão}

A investigação de depressão foi feita mediante anamnese padronizada da instituição. Conforme item A dos critérios diagnósticos do DSM-IV da American Psychiatric Association 11, o diagnóstico de depressão foi feito no caso de ocorrência de cinco (ou mais) determinados sintomas, com comprometimento funcional, durante período mínimo de duas semanas, sendo pelo menos um dos sintomas humor deprimido ou perda de interesse/prazer. Os demais sintomas são: (a) perda ou ganho de peso; (b) insônia ou excesso de sono; (c) agitação ou retardo psicomotor; (d) fadiga ou perda de energia; (e) sentimento de inutilidade ou culpa excessiva; (f) dificuldade de concentração; (g) pensamento de morte recorrente ou idéias suicidas.

\section{Avaliação da comorbidade crônica}

Foram estudadas 14 doenças clínicas, cujos critérios diagnósticos foram os seguintes:

a) hipertensão arterial: pressão sistólica $\geq$ $140 \mathrm{mmHg}$ e/ou pressão diastólica $\geq 90 \mathrm{mmHg}$, aferida em duas ocasiões; ou uso atual de antihipertensivos;

b) diabetes mellitus: glicemia de jejum $\geq 126 \mathrm{mg}$ / dl (em mais de uma ocasião) ou curva glicêmica maior que 200mg/dl (em dois momentos); ou uso atual de hipoglicemiantes;

c) acidente vascular cerebral (AVC): relato de AVC prévio, objetivamente comprovado por exame físico, exame de imagem ou relatório de alta hospitalar;

d) osteoartrose: presença de manifestações clínicas da doença (artralgia, deformidade articular e limitação funcional);

e) incontinência urinária: perda involuntária de qualquer quantidade de urina;

f) obstipação intestinal: freqüência menor que três evacuações por semana;

g) doença de Parkinson: presença de dois sinais da tétrade clássica (tremor de repouso, bradicinesia, rigidez e instabilidade postural), sendo o diagnóstico firmado pelo Núcleo de Apoio ao Paciente com Parkinson (NAPP) do mesmo ambulatório;

h) dislipidemia: colesterol total $\geq 240 \mathrm{mg} / \mathrm{dl}$, colesterol-LDL $\geq 160$ e/ou triglicérides $\geq 200 \mathrm{mg} / \mathrm{dl}$; i) instabilidade postural: relato de quedas freqüentes e/ou instabilidade postural comprovada ao exame físico;

j) cardiopatias: relato de cardiopatia, objetivamente comprovado por exame físico, laudos de exames ou relatório médico;

k) demência: comprometimento da memória associado a déficit de outra função cognitiva, 
com prejuízo funcional e social, não caracterizado como delirium;

l) doença pulmonar obstrutiva crônica (DPOC): sinais clínicos e/ou exames complementares compatíveis com bronquite crônica, enfisema pulmonar ou asma;

m) hipotireoidismo: níveis de TSH acima dos valores de referência;

n) câncer: diagnóstico atual ou história prévia de neoplasia, objetivamente comprovado por laudo de exames complementares e/ou reclatório médico.

\section{Análise dos dados}

A variável dependente foi o diagnóstico de depressão; as variáveis independentes foram as 14 patologias supracitadas (dicotômicas) e o número de patologias crônicas. As co-variáveis de interesse foram: sexo, idade e estado nutricional. Após a análise descritiva das variáveis, foi calculada a razão de prevalência (RP) bruta, com intervalo de confiança (IC) de 95\%, para cada patologia de interesse. Por fim, foi realizada análise estratificada, por sexo, por idade e por estado nutricional.

\section{Resultados}

A população de estudo foi constituída de 1.120 idosos, cuja idade variou de 60 a 99 anos, com média igual a 75,4 $( \pm 7,7)$ anos. Aproximadamente metade dos indivíduos estava na faixa de 7079 anos. As mulheres representaram $72,5 \%$ dos sujeitos estudados. Não houve diferença significativa na distribuição por sexo entre as diversas faixas etárias $\left(\chi^{2}=0,75 \mathrm{p}=0,86\right)$. O IMC variou de 13,3 a $56,2 \mathrm{~kg} / \mathrm{m}^{2}$, com média igual a $25,1(+4,6)$ $\mathrm{kg} / \mathrm{m}^{2}$. A desnutrição e a obesidade estiveram presentes respectivamente em $19 \%$ e em $26,2 \%$ da população (Tabela 1).

As doenças clínicas mais freqüentes foram hipertensão arterial, osteoartrose e incontinência urinária, seguidas por dislipidemia, instabilidade postural e diabetes mellitus (Tabela 2). O número de patologias de cada indivíduo variou de 0 a 8 , com mediana igual a 3. A grande maioria $(91 \%)$ apresentava entre um e cinco problemas crônicos. Cinqüenta e nove idosos $(5,3 \%)$ não apresentaram qualquer um dos problemas estudados (Tabela 3).

A depressão foi diagnosticada em 262 indivíduos $(23,4 \%)$, tendendo a ser mais freqüente entre os menores de 75 anos $(\mathrm{RP}=1,24$; IC95\%: $1,00-1,53)$ e entre as mulheres $(\mathrm{RP}=1,28$; IC95\%: 0,99-1,65), com significância estatística entre as menores de 80 anos ( $R P=1,39$; IC95\%: 1,03-1,90). Não houve associação da depressão com o estado nutricional.
Tabela 1

Distribuição dos indivíduos segundo variáveis demográficas e estado nutricional. Salvador, Bahia, Brasil, 2001-2004.

\begin{tabular}{lcc}
\hline Variáveis & $\mathbf{n}$ & $\%$ \\
\hline Sexo & & \\
$\quad$ Feminino & 812 & 72,5 \\
$\quad$ Masculino & 308 & 27,5 \\
$\quad$ Total & 1.120 & \\
Faixa etária (anos) & & \\
60-69 & 272 & 24,3 \\
70-79 & 533 & 47,6 \\
80-89 & 272 & 24,3 \\
$>$ 90 & 43 & 3,8 \\
Total & 1.120 & \\
Estado nutricional & & \\
Desnutrição (IMC <22) & 198 & 19,0 \\
Eutrofia (IMC = 22-27) & 572 & 54,8 \\
Obesidade (IMC > 27) & 273 & 26,2 \\
Total & 1.043 & \\
\end{tabular}

$\mathrm{IMC}=$ índice de massa corporal $\left(\mathrm{kg} / \mathrm{m}^{2}\right)$.
Distribuição dos agravos da saúde em idosos $(n=1.120)$.

Salvador, Bahia, Brasil, 2001-2004.

\begin{tabular}{lcc}
\hline Patologias & $\mathbf{n}$ & $\%$ \\
\hline Hipertensão arterial & 697 & 62,2 \\
Osteoartrose & 448 & 40,0 \\
Incontinência urinária & 394 & 35,2 \\
Dislipidemia & 261 & 23,3 \\
Instabilidade postural & 249 & 22,2 \\
Diabetes mellitus & 197 & 17,6 \\
Obstipação intestinal & 176 & 15,7 \\
Cardiopatias & 137 & 12,2 \\
Demência & 135 & 12,1 \\
Acidente vascular cerebral & 95 & 8,5 \\
Doença de Parkinson & 68 & 6,1 \\
Doença pulmonar obstrutiva crônica & 59 & 5,3 \\
Hipotireoidismo & 50 & 4,5 \\
Câncer & 25 & 2,2 \\
\hline
\end{tabular}

Observou-se associação positiva entre depressão e número de patologias crônicas maior que três. A doença de Parkinson foi a patologia que mais se associou com a depressão (Tabela 4), alcançando significância estatística no sexo feminino ( $R P=1,59$; IC95\%: 1,05-2,41) e na faixa etária de 70-79 anos (RP = 2,02; IC95\%: 1,28-3,20); 
Tabela 3

Distribuição dos idosos segundo o número de patologias.

Salvador, Bahia, Brasil, 2001-2004.

\begin{tabular}{lccc}
\hline Patologias & $\mathbf{n}$ & $\%$ & \% cumulativo \\
\hline Nenhuma & 59 & 5,3 & 5,3 \\
1 & 178 & 15,9 & 21,2 \\
2 & 316 & 28,2 & 49,4 \\
3 & 269 & 24,0 & 73,4 \\
4 & 174 & 15,5 & 88,9 \\
5 & 82 & 7,3 & 96,3 \\
6 & 28 & 2,5 & 98,8 \\
7 & 12 & 1,1 & 99,8 \\
8 & 2 & 0,2 & 100,0 \\
\hline
\end{tabular}

Tabela 4

Associação entre depressão e doenças clínicas em idosos.

Salvador, Bahia, Brasil, 2001-2004.

\begin{tabular}{lcc}
\hline Patologias & RP & IC95\% \\
\hline Doença de Parkinson & 1,42 & $0,99-2,04$ \\
Obstipação intestinal & 1,27 & $0,97-1,65$ \\
Instabilidade postural & 1,20 & $0,95-1,53$ \\
Câncer & 1,20 & $0,64-2,28$ \\
Incontinência urinária & 1,14 & $0,92-1,41$ \\
Osteoartrose & 1,10 & $0,89-1,37$ \\
Acidente vascular cerebral & 1,09 & $0,76-1,56$ \\
Doença pulmonar obstrutiva crônica & 1,02 & $0,63-1,62$ \\
Cardiopatias & 0,96 & $0,69-1,34$ \\
Dislipidemia & 0,94 & $0,72-1,21$ \\
Diabetes & 0,92 & $0,69-1,23$ \\
Hipertensão arterial & 0,85 & $0,69-1,06$ \\
Hipotireoidismo & 0,85 & $0,48-1,49$ \\
Demência & 0,74 & $0,50-1,08$ \\
N (patologias) > 3 & 1,31 & $1,04-1,66$ \\
\hline
\end{tabular}

nesse caso, além da doença de Parkinson, associaram-se positivamente à depressão a incontinência urinária ( $\mathrm{RP}=1,45$; IC95\%: 1,05-1,98), a instabilidade postural $(\mathrm{RP}=1,71$; IC95\%: 1,23 $2,37)$ e a obstipação intestinal $(\mathrm{RP}=1,72$; IC95\%: $1,22-2,24)$.

As diferenças encontradas entre os estratos etários apresentaram significância estatística no tocante à associação de depressão com instabilidade postural $\left(\chi^{2}=10,3 ; p=0,006\right)$, obstipação intestinal $\left(\chi^{2}=6,28 ; p=0,043\right)$ e número de doenças crônicas maior que três $\left(\chi^{2}=6,73 ; p=0,035\right)$. A associação entre hipertensão arterial e depressão diferiu significativamente por sexo, tornando-se positiva no sexo masculino $\left(\chi^{2}=4,51 ; p=0,033\right)$. Entre os obesos, evidenciou-se associação entre instabilidade postural e depressão $\left(\chi^{2}=6,99 ; \mathrm{p}=\right.$ $0,03)$, não observada nos demais estratos nutricionais. Não se observaram outras diferenças por sexo, idade ou estrato nutricional.

\section{Discussão}

O presente trabalho chama a atenção para a presença de depressão em idosos portadores de doenças crônicas, os quais muitas vezes não são atendidos por geriatras nem por psiquiatras, profissionais obviamente treinados para a investigação da sintomatologia depressiva. O envelhecimento populacional fará com que os médicos e profissionais de saúde, independentemente de sua especialidade, lidem cada vez mais com pacientes idosos.

A preponderância do sexo feminino na população estudada reflete não só o maior percentual de mulheres na terceira idade, como também a maior procura dos serviços de saúde por parte destas 12. A PNAD (2004) 4 evidenciou que a parcela feminina representa $56 \%$ do contingente de idosos.

A média de idade pode ser conseqüência dos critérios de triagem do centro, no qual idade maior ou igual a 75 anos garante matrícula irrestrita dos idosos, ao passo que o acesso dos idosos mais jovens só se faz na presença de uma síndrome geriátrica. Metade da população estudada estava na faixa etária de 70 a 79 anos, enquanto no Estado da Bahia, em 2004, os indivíduos desse grupo etário correspondiam a $30 \%$ da população idosa (DATASUS, http:/ /www.datasus.gov.br).

A freqüência de desnutrição foi semelhante à observada em idosos brasileiros acompanhados ambulatorialmente: cerca de 20\% 10 . Com base nos dados da Pesquisa Nacional sobre Saúde e Nutrição (PNSN), Tavares \& Anjos 13 demonstraram que a prevalência de magreza na população idosa brasileira é de 7,8\%, mais freqüente em mulheres nas faixas etárias mais avançadas, com diferenças regionais, como em áreas rurais do Nordeste, onde a desnutrição alcançou 17,6\% das mulheres idosas.

A freqüência de obesidade aproxima-se da encontrada em um estudo multicêntrico em ambulatórios de cardiologia e geriatria de instituições brasileiras, segundo o qual 30\% dos idosos avaliados apresentavam obesidade 14 , resultado semelhante ao da PNSN 13. A menor freqüência de obesidade entre os mais idosos é condizente com a literatura, que afirma ser a obesidade menos freqüente em pessoas com mais de 75 
anos 10. A diferença por gênero encontrada no presente estudo também era esperada, pois a obesidade, na velhice, é mais prevalente no sexo feminino, acometendo $37 \%$ das mulheres e $23 \%$ dos homens idosos na Região Nordeste do Brasil 13 .

O diagnóstico de depressão foi feito em $23,4 \%$ dos idosos avaliados, o que está dentro da ampla faixa citada na literatura 15. É importante ressaltar a grande freqüência de depressão em um serviço de atenção ao idoso: praticamente um em cada quatro pacientes preencheu o item A dos critérios do DSM-IV, para depressão maior, sem contar aqueles que cursam com sintomatologia depressiva, mas não preenchem tais critérios e, por isso, não foram considerados neste trabalho.

Uma vez que a síndrome depressiva é um dos critérios de triagem do centro de referência, a alta freqüência de depressão pode se dever a um viés de seleção. Seria interessante verificar quantos pacientes já ingressaram com o diagnóstico de depressão, já que apenas 30\% a 50\% dos pacientes deprimidos são diagnosticados por seus médicos 15,16,17,18.

A geriatria, por trabalhar com a abordagem multidimensional e, na maioria das vezes, com equipe multidisciplinar, é possivelmente a especialidade clínica que mais se preocupa com a investigação da sintomatologia depressiva. Infelizmente, trata-se de uma especialidade relativamente jovem, e o número de profissionais de saúde com treinamento na área é muito pequeno em face do contingente de idosos. Na prática, o idoso, que na maioria das vezes é portador de múltiplas patologias crônicas, termina sendo avaliado por diversos especialistas, e estes, focados no seu alvo de abordagem, perdem a visão integral do indivíduo, deixando de diagnosticar e tratar doenças que interagem entre si, com efeitos deletérios sobre a saúde e a qualidade de vida dos seus pacientes.

O uso rotineiro, por parte dos clínicos, de instrumentos de triagem de depressão, facilmente aplicáveis na prática clínica, permitiria não só o diagnóstico de muitos casos da doença, que passam despercebidos e influenciam adversamente a qualidade de vida dos pacientes, como também o prognóstico das comorbidades existentes, as quais contribuem para uma maior mortalidade, seja pelo aumento do risco de suicídio, seja pela evolução desfavorável das doenças crônicas existentes 8,19,20,21,22.

A depressão é uma doença, e não uma manifestação do envelhecimento fisiológico; portanto, necessita ser diagnosticada e tratada. Como lembra Solomon 23 (p. 175), "a suposição de que é lógico os velhos serem infelizes nos impede de tratar essa infelicidade, deixando muita gente vi- vendo seus últimos dias numa extrema e desnecessária dor emocional".

No presente estudo, o sexo feminino e a idade menor que 75 anos associaram-se com depressão. Enquanto a diferença por gênero é bem documentada na literatura mundial 8,23 , não existe consenso sobre a faixa etária de maior prevalência de depressão; alguns autores afirmam que ocorre mais entre os mais idosos, ao passo que outros demonstram justamente o contrário 15.

Embora envelhecer não implique adoecer, os idosos são, na grande maioria, portadores de doenças crônicas e múltiplas. Em 1999, um estudo transversal com amostra aleatória de idosos residentes nos Estados Unidos demonstrou que 82\% deles tinham pelo menos uma doença crônica e $65 \%$ tinham múltiplas doenças crônicas 24 . Logo, a comorbidade em idosos passa a ser mais a regra do que a exceção, possibilitando, assim, uma gama de interações entre as diversas patologias, com repercussões orgânicas e psicossociais.

Na literatura mundial, a prevalência de sintomas depressivos tem sido fortemente associada com o número de doenças crônicas, mesmo após ajuste das possíveis variáveis de confundimento 25,26 . Tem-se documentado amplamente, por exemplo, que a depressão é a principal variável que influencia negativamente a qualidade de vida dos pacientes parkinsonianos 27,28,29,30,31 .

No presente estudo, ficou evidenciado que o idoso portador de mais de três patologias crônicas cursa mais freqüentemente com sintomatologia depressiva. Corroborando a literatura mundial 9 , os indivíduos parkinsonianos apresentaram até duas vezes mais depressão que os demais.

Dessa forma, considerando que a depressão antecede os sintomas parkinsonianos em alguns pacientes 27 , estudos devem ser conduzidos com a finalidade de investigar o papel da depressão como fator de risco para o desenvolvimento da doença de Parkinson. Além disso, as equipes que atendem os pacientes parkinsonianos devem ser treinadas adequadamente para a investigação e o manejo da síndrome depressiva, em virtude da maior prevalência de depressão e do seu papel como fator prognóstico na doença de Parkinson.

Por sua vez, a instabilidade postural acarreta o risco de queda, mas as seqüelas físicas e psicossociais da redução excessiva dos movimentos podem ser mais deletérias do que a queda propriamente dita 32 . Uma vez que o comprometimento funcional é associado com prevalência de depressão até seis vezes maior 33, é possível que a associação entre depressão e instabilidade postural se deva ao comprometimento funcional, o que não foi controlado no presente trabalho. $\mathrm{O}$ mesmo se aplica à incontinência urinária que é causa de estigmatização, isolamento social e sin- 
tomatologia depressiva 34,35 , sendo que os idosos incontinentes podem apresentar-se deprimidos até duas vezes mais freqüentemente que os continentes.

Já a associação entre obstipação intestinal e depressão pode ser analisada de modo bidirecional: os indivíduos portadores de obstipação intestinal crônica ao longo da vida poderiam ser maiores candidatos a desenvolver depressão e/ou os indivíduos deprimidos podem ter maior freqüência de obstipação intestinal relacionada aos hábitos de vida (ingesta reduzida e inatividade física) e/ou por efeito adverso dos antidepressivos.

Todas as associações entre depressão e morbidade crônica discutidas acima apresentaram significância estatística, mas é importante destacar outras associações encontradas no presente estudo: com câncer, osteoartrose, AVC e DPOC. Diversos estudos têm documentado a associação entre depressão e câncer 16,36,37. Depressão é freqüente entre os portadores de artrite e o tratamento antidepressivo promove melhora da capacidade funcional e da qualidade de vida desses pacientes 37 . Revisões sistemáticas têm destacado o caráter bidirecional da associação entre depressão e AVC 38,39: de um lado, a ocorrência de um AVC aumenta o risco de e, por outro lado a depressão pode ser fator de risco para a ocorrência de AVC. Pacientes com DPOC grave têm risco duas vezes maior de depressão que os controles, após ajuste das variáveis de confundimento 40. Todas essas possíveis associações encontram respaldo na literatura mundial.

Curiosamente, não foi observada associação entre depressão e diabetes mellitus. De acordo com uma meta-análise realizada em 2001 20, há o dobro de chance de depressão maior em pacientes diabéticos do que em pacientes sem diabetes mellitus, embora os estudos não se restrinjam a idosos. Um grande inquérito populacional, recentemente publicado, evidenciou que pacientes com diabetes tipo 2 sem outras doenças crônicas têm a mesma chance de depressão que a população não diabética, diferentemente dos diabéticos tipo 1 , nos quais a comorbidade crônica não se correlacionou com a depressão ${ }^{41}$.

Além do diagnóstico de depressão maior, existem outras formas de apresentação de depressão que cursam com sintomas menos intensos, porém com grau de incapacidade semelhante, como, por exemplo, distimia, transtorno misto e depressão subsindrômica ${ }^{18}$. O presente estudo considerou apenas os casos de depressão maior, cujos critérios diagnósticos estão bem estabelecidos. É possível que a exclusão dos outros casos de sintomas depressivos seja responsável em parte pela não detecção de algumas associações esperadas.
Em estudo semelhante ao atual, realizado em um ambulatório geriátrico na Turquia ${ }^{42}$, a depressão esteve presente em 21,8\% dos idosos avaliados $(\mathrm{n}=1.255)$. Entre as nove patologias crônicas investigadas, três estiveram associadas com depressão: hipertensão arterial, demência e osteoporose. O trabalho não faz qualquer referência à doença de Parkinson, incontinência urinária nem instabilidade postural, mesmo tendo sido realizado em um ambulatório geriátrico de referência.

Bisschop et al. 26, acompanhando 2 mil idosos durante seis anos, em Amsterdam, demonstraram associação entre sintomas depressivos e AVC, doença pulmonar, artrite, doença cardíaca e câncer; o comprometimento funcional foi um preditor importante de depressão nos pacientes com AVC, mas não nos demais 26 . O presente trabalho não avaliou o comprometimento funcional como variável preditora de depressão, o que seria interessante em estudos posteriores.

Quanto às doenças crônicas, embora este estudo tenha sido realizado em um centro de referência com amostra de conveniência, as freqüências encontradas não são discrepantes em relação aos levantamentos populacionais 19,43,44,45 e se assemelham também àquelas encontradas em nível ambulatorial 14,42,46. Por exemplo, observaram-se diferenças significativas por faixa etária quanto à freqüência de algumas doenças crônicas estudadas, tendo havido maior freqüência de demência entre os mais idosos, o que está de acordo com a literatura. Em um estudo transversal de base populacional, foi observado que a prevalência de demência aumenta de 0,53\%, na faixa etária de 55-59 anos, até $43,2 \%$, no grupo de 95 anos ou mais 47. Porém, contrariando o pensamento de muitos de que a demência é inevitável para quem ultrapassa os 100 anos, estudos com centenários mostram que em torno de $15 \%$ a $25 \%$ destes se mantêm funcionalmente intactos do ponto de vista cognitivo. Entre os demais, o início do comprometimento cognitivo evidente só ocorreu após os 92 anos 48 .

A incontinência urinária também foi mais freqüente entre os mais idosos, o que é consistente com os dados encontrados em outras pesquisas. Ko et al. 35 , estudando uma grande amostra aleatória de idosos, demonstraram que $25 \%$ dos sujeitos tinham dificuldade no controle urinário, acometendo $30 \%$ dos maiores de 75 anos.

A menor freqüência de hipertensão e de dislipidemia entre os mais idosos, no presente estudo, pode refletir a mortalidade cardiovascular mais precoce, bem como a mudança de hábitos alimentares e a redução de peso com o avançar da idade. Segundo dados do estudo de Framingham, a hipercolesterolemia nos idosos é mais 
freqüente na faixa etária de 65 a 74 anos, declinando gradativamente após os 75 anos 49 .

A sobrevida encurtada dos pacientes parkinsonianos deve ser responsável pela maior freqüência de doença de Parkinson no estrato mais jovem, o dobro da encontrada nos demais grupos etários. A mortalidade desses pacientes permanece superior à esperada, apesar dos avanços terapêuticos, podendo estar associada a fraturas de colo de fêmur, pneumonias, doença cardiovascular e delirium 50 .

As diferenças por gênero na prevalência das diversas doenças crônicas evidenciadas neste trabalho também encontram respaldo na literatura: enquanto o sexo feminino associou-se positivamente com osteoartrose, dislipidemia e incontinência urinária 49,51,52,53,54, o sexo masculino associou-se com AVC, cardiopatias, doença de Parkinson e DPOC 6,29,46,52.

Os dados antropométricos não foram mensurados em 77 indivíduos. A análise da morbidade crônica evidenciou, nesse grupo, maior freqüência de patologias que se associam com dependência funcional (demência, AVC, doença de Parkinson, incontinência urinária e instabilidade postural), o que pode ter dificultado ou impossibilitado a mensuração do peso e altura. A maior freqüência de obstipação intestinal nesse subgrupo pode ser conseqüência da restrição da mobilidade, imposta por tais patologias.

A impossibilidade de estabelecer uma seqüência temporal entre as associações encontradas é uma das limitações deste trabalho por se tratar de um estudo transversal. A origem da população (centro de referência) impossibilita as generalizações para a população como um todo. O centro de referência tende a reunir maior número de casos de depressão e de portadores de doenças crônicas, o que pode ter levado a vieses de seleção.

Por outro lado, é possível que a prevalência de algumas condições crônicas, como AVC, cardio- patias e câncer, tenham sido subestimadas em virtude das limitações dos meios diagnósticos, já que o critério diagnóstico não repousou apenas no relato do paciente, mas na comprovação objetiva dessas entidades nosológicas, o que muitas vezes depende de recursos tecnológicos escassos no Sistema Único de Saúde.

A amostra de conveniência constituída por pacientes atendidos seqüencialmente por um único examinador restringe a generalização para os pacientes do centro, limitando, em parte, a validade interna do trabalho. Não foi possível compor uma amostra aleatória por falta de uniformização dos registros, com muitos dados incompletos ou ausentes nos prontuários. Algumas variáveis passíveis de confundimento não foram controladas neste estudo, entre as quais: estado civil, história ocupacional, condições de moradia, composição domiciliar, renda familiar, tabagismo, etilismo e comprometimento funcional.

A falta de conhecimento do perfil sóciodemográfico e do perfil de morbidade da população assistida dificulta o planejamento e a avaliação do desempenho de serviços e equipes de saúde. Sabe-se que, para obter tal conhecimento, a opção mais adequada é a realização de inquéritos de base populacional, entretanto isso nem sempre é possível, exigindo recursos humanos e financeiros de relativa magnitude. Uma alternativa bastante utilizada são os estudos com base em demanda a serviços de saúde, que podem fornecer informações valiosas, com custos bastante reduzidos, como é o caso do presente estudo.

Não obstante as limitações existentes, este trabalho ressalta a importância da abordagem multidimensional do idoso, considerando que a comorbidade nessa população é mais a regra do que a exceção, e que a associação entre depressão e doença clínica poderá oferecer dificuldades tanto no diagnóstico, como no manejo de ambas. 


\section{Resumo}

Este estudo investiga a associação entre depressão e comorbidade clínica em idosos em Salvador, Bahia, Brasil. A população estudada consistiu de uma amostra de idosos $(n=1.120)$ atendidos em um ambulatório de referência. Razões de prevalência brutas entre depressão e doenças clínicas foram calculadas, com subseqüente estratificação por sexo, faixa etária e estado nutricional. Noventa e cinco por cento dos indivíduos apresentavam pelo menos uma patologia crônica, sendo as principais: hipertensão arterial $(62,2 \%)$, osteoartrose (40\%) e incontinência urinária (35\%). A depressão foi diagnosticada em $23,4 \%$, sendo mais freqüente entre as mulheres $(R P=1,28$; IC95\%: 0,99-1,65) $e$ entre os menores de 75 anos ( $R P=1,24$; IC95\%: 1,00-1,53). O número de doenças crônicas maior que três foi associado com depressão ( $R P=1,31$; IC95\%: 1,04-1,66). Observou-se associação, ainda, entre depressão e doença de Parkinson, sobretudo no sexo feminino $(R P=1,59$; IC95\%: 1,05-2,41) e na faixa etária de 70-79 anos (RP = 2,02; IC95\%: 1,28-3,20). Como muitos idosos apresentam doenças crônicas e múltiplas, os profissionais de saúde devem estar atentos para a possibilidade de sintomatologia depressiva nesses pacientes.

Idoso; Saúde do Idoso; Doença Crônica; Depressão

\section{Referências}

1. Kalache A, Veras RP, Ramos LR. O envelhecimento da população mundial: um desafio novo. Rev Saúde Pública 1987; 21:200-10

2. Ramos LR, Veras RP, Kalache A. Envelhecimento populacional: uma realidade brasileira. Rev Saúde Pública 1987; 21:211-24.

3. Veras RP, Ramos LR, Kalache A. Crescimento da população idosa no Brasil: transformações e conseqüências na sociedade. Rev Saúde Pública 1987; 21:225-33.

4. Instituto Brasileiro de Geografia e Estatística. Pesquisa Nacional por Amostras de Domicílios 2004. http://www.ibge.gov.br (acessado em 31/ Out/2005).

5. Veras RP. Em busca de uma assistência adequada à saúde do idoso: revisão de literatura. Cad Saúde Pública 2002; 19:705-15.

6. Lessa I. Epidemiologia das doenças crônicas nãotransmissíveis. São Paulo: Editora Hucitec; 1998.

\section{Colaboradores}

M. B. Duarte elaborou o artigo em todas as suas etapas. M. A. V. Rego contribuiu na análise estatística e na revisão da redação final do artigo aprovado para publicação.
7. Holman H. Chronic disease - the need for a new clinical education. JAMA 2004; 292:1057-9.

8. Serby M, Yu M. Overview: depression in the elderly. Mt Sinai J Med 2003; 70:38-44.

9. Alexopoulos GS, Buckwalter K, Olin J, Martinez R, Wainscott C, Krishnan KRR. Comorbidity of late life depression: an opportunity for research on mechanisms and treatment. Biol Psychiatry 2002; 52:543-58.

10. Najas M, Pereira FAI. Nutrição. In: Freitas EV, Py L, Nery AL, Cançado FAX, Gorzoni ML, Rocha SM, organizadores. Tratado de geriatria e gerontologia. Rio de Janeiro: Editora Guanabara Koogan; 2002. p. 838-45.

11. American Psychiatric Association. Diagnostic and statistical manual of mental disorders - DSM-IV. 4th Ed. Washington DC: American Psychiatric Association; 1994 
12. Camarano AA. Envelhecimento da população brasileira: uma contribuição demográfica. In: Freitas EV, Py L, Nery AL, Cançado FAX, Gorzoni ML, Rocha SM, organizadores. Tratado de geriatria e gerontologia. Rio de Janeiro: Editora Guanabara Koogan; 2002. p. 58-71.

13. Tavares EL, Anjos LA. Perfil antropométrico da população idosa brasileira. Resultados da Pesquisa Nacional sobre Saúde e Nutrição. Cad Saúde Pública 1999; 15:759-68.

14. Tadei CFG, Ramos LR, De Morais JC, Wajngarten M, Libberman A, Santos SC, et al. Estudo multicêntrico de idosos atendidos em ambulatórios de cardiologia e geriatria de instituições brasileiras. Arq Bras Cardiol 1997: 69:327-33.

15. Alexopoulos GS, Borson S, Cuthbert BN, Devanand PN, Mulsant BH, Olin JT, et al. Assessment of late life depression. Biol Psychiatry 2002; 52:164-74.

16. Charlson M, Peterson JC. Medical comorbidity and late life depression: what is known and what are the unmet needs? Biol Psychiatry 2002; 52:226-35.

17. Gaynes BN, Burns BJ, Tweed DL, Erickson P. Depression and health-related quality of life. J Nerv Ment Dis 2002; 190:799-806.

18. Fleck MPA, Lafer B, Sougey EB, Del Porto JA, Brasil MA, Juruena MF. Diretrizes da Associação Médica Brasileira para o tratamento da depressão. Rev Bras Psiquiatria 2003; 25:114-22.

19. Black AS, Markides KS. Depressive symptoms and mortality in older Mexican Americans. Ann Epidemiol 1999; 9:45-52.

20. Anderson RJ, Freedland KE, Clouse RE, Lustmans PJ. The prevalence of comorbid depression in adults with diabetes: a meta-analysis. Diabetes Care 2001; 24:1069-78.

21. Takeshita J, Masaki K, Ahmed I, Foley DJ, Li YQ, Chen R, et al. Are depressive symptoms a risk factor for mortality in elderly Japanese American Men? The Honolulu-Asia aging study. Am J Psychiatry 2002; 159:1127-32.

22. Unützer J. Diagnosis and treatment of older adults with depression in primary care. Biol Psychiatry 2002; 52:285-92.

23. Solomon A. O demônio do meio-dia - uma anatomia da depressão. Rio de Janeiro: Editora Objetiva; 2002.

24. Wolff JL, Starfield B, Anderson G. Prevalence, expenditures and complications of multiple chronic conditions in the elderly. Arch Intern Med 2002; 162:2269-76

25. Mills TL. Comorbid depressive symptomatology: isolating the efects of chronic medical conditions on self-reported depressive symptoms among community-dwelling older adults. Soc Sci Med 2001; 53:569-78.

26. Bisschop MI, Kriegsman DMW, Deeg DJH, Beekman ATF, Tilburg W. The longitudinal relation between chronic diseases and depression in older persons in the community: the Longitudinal Aging Study Amsterdam. J Clin Epidemiol 2004; 57: 187-94.

27. Silberman CD, Laks J, Rodrigues CS, Engelhardt E. Uma revisão sobre depressão como fator de risco na doença de Parkinson e seu impacto na cognição. Rev Psiquiatr Rio Gd Sul 2004; 2:52-60.
28. Karlsen KH, Larsen JP, Tandberg E, Mæland JG. Influence of clinical and demographic variables on quality of life in patients with Parkinson's disease. J Neurol Neurosurg Psychiatr 1999; 66:431-5.

29. Hobson P, Holden A, Meara J. Measuring the impact of Parkinson's disease with the Parkinson's disease quality of life questionnaire. Age Ageing 1999; 28:341-6.

30. Schrag A, Jahanshahi M, Quinn N. What contributes to quality of life in patients with Parkinson's disease? J Neurol Neurosurg Psychiatr 2000; 69:308-12.

31. Gage H, Hendricks A, Zhang S, Kazis L. The relative health related quality of life of veterans with Parkinson's disease. J Neurol Neurosurg Psychiatr 2003; 74:163-9.

32. Studenski S. Quedas. In: Calkins E, Ford AB, Katz PR, organizadores. Geriatria prática. Rio de Janeiro: Editora Revinter; 1997. p. 227-33.

33. Heikkinen RL, Kauppinen M. Depressive symptoms in late life: a 10-year follow-up. Arch Gerontol Geriatr 2004; 38:239-50.

34. Tannenbaum C, Perrin L, DuBeau CE, Kuchel GA. Diagnosis and management of urinary incontinence in the older patient. Arch Phy Med Rehabil 2001; 82:134-8.

35. Ko Y, Lin SJ, Salmon JW, Bron MS. The impact of urinary incontinence on quality of life of the elderly. Am J Manag Care 2005; 11(4 Suppl):S103-11.

36. Krishnan KRR. Biological risk factors in late life depression. Biol Psychiatry 2002; 52:185-92.

37. Chapman DP, Perry GS, Strine TW. The vital link between chronic disease and depressive disorders. Prev Chronic Dis 2005; 2:A33.

38. Terroni LMN, Leite CC, Tinone G, Fráguas Jr. R. Depressão pós-AVC: fatores de risco e terapêutica antidepressiva. Rev Assoc Méd Bras 2003; 49: 450-9.

39. Ramasubbu R, Patten SB. Effect of depression on stroke morbidity and mortality. Can J Psychiatry 2003; 48:250-7.

40. Manen JG, Bindels PJE, Dekker FW, Jzermans CJI, Zee JS, Schadé E. Risk of depression in patients with chronic obstructive pulmonary disease and its determinants. Thorax 2002; 57:412-6.

41. Engum A, Mykletum A, Midthjell K, Holen A, Dahl AA. Depression and diabetes: a large populationbased study of sociodemographic, lifestyle and clinical factors associated with depression in type 1 and type 2 diabetes. Diabetes Care 2005; 28 : 1904-9.

42. Cankurtaran M, Halil M, Yavuz BB, Dagli N, Cankurtaran ES, Ariogul S. Depression and concomitant diseases in a Turkish geriatric outpatient setting. Arch Gerontol Geriatr 2005; 40:307-15.

43. Cacciatore F, Gallo C, Ferrara N, Abete P, Paolisso G, Canonico S, et al. Morbidity patterns in aged population in southern Italy. A survey sampling. Arch Gerontol Geriatr 1998; 26:201-13.

44. Corrales D, Palomo L, Bravo MJM, Alonso G, Torrico P, Barroso A, et al. Capacidad funcional y problemas socioasistenciales de los ancianos del área de salud de Cáceres. Aten Primaria 2004; 33 . 426-33. 
45. Feliciano AB, Moraes SA, Freitas ICM. O perfil do idoso de baixa renda no Município de São Carlos, São Paulo, Brasil: um estudo epidemiológico. Cad Saúde Pública 2004; 20:1575-85.

46. Saks K, Kolk H, Allev R, Soots A, Koiv K, Paju I, et al. Health status of the older population in Estonia. Croat Med J 2001; 42:663-8.

47. Ott A, Breteler MMB, van Harskamp F, Claus JJ, van der Cammen TJM, Grobbee DE, Hofman A. Prevalence of Alzheimer's disease and vascular dementia: association with education. The Rotterdam study. BMJ 1995; 310:970-3.

48. Perls T. Centenarians who avoid dementia. Trends Neurosci 2004; 27:633-6.

49. Moriguchi EH, Michelon E, Vieira JLC. Dislipidemia em idosos. In: Freitas EV, Py L, Nery AL, Cançado FAX, Gorzoni ML, Rocha SM, organizadores. Tratado de geriatria e gerontologia. Rio de Janeiro: Editora Guanabara Koogan; 2002. p. 239-48.
50. Fernandez HH, Lapane KL. Predictors of mortality among nursing home residents with a diagnosis of Parkinson's disease. Med Sci Monit 2002; 8:241-6.

51. De Filippis S, Gulli A, Caliri A, Romano C, Munao F, Trimarchi G, et al. Epidemiologia e fattori di rischio dell'osteoartrosi: review della letteratura e dati dal progetto OASIS. Reumatismo 2004; 56:169-84.

52. Lima-Costa MF, Barreto SM, Giatti L. Condições de saúde, capacidade funcional, uso de serviços de saúde e gastos com medicamentos da população idosa brasileira: um estudo descritivo baseado na Pesquisa Nacional por Amostra de Domicílios. Cad Saúde Pública 2003; 19:735-43.

53. Durrant J, Snape J. Urinary incontinence in nursing homes for older people. Age Ageing 2003; 32:12-8.

54. Patel M, Coshall C, Rudd AG, Wolfe CDA. Natural history and effects on 2-year outcomes of urinary incontinence after stroke. Stroke 2001; 32:122-7.

Recebido em 07/Dez/2005

Versão final reapresentada em 22/Mai/2006

Aprovado em 10/Jul/2006 